\title{
Apport de la dixième révision de la Classification internationale des maladies dans le codage de la mortalité médico-légale
}

Ons Hmandi, ${ }^{1,2,}$ Mehdi Ben Khelil, ${ }^{1,2,}$ Nouha Zoghlami, ${ }^{3}$ Hajer Skhiri ${ }^{1,3}$ and Moncef Hamdoun ${ }^{1,2}$

${ }^{1}$ Faculté de Médecine de Tunis, Université El Manar, Tunisie. ${ }^{2}$ Service de médecine légale, Hôpital Charles Nicolle de Tunis, Tunisie. ${ }^{3}$ Institut National de Santé Publique, Tunisie. * Partagent la première position ( Correspondance à adresser à : benkhelilmehdi@yahoo.fr ).

\section{Résumé}

Contexte : les rapports de mortalité en Tunisie n'incluent pas les causes de décès médico-légaux.

Objectif : établir les statistiques sur les causes de décès médico-légaux au nord de la Tunisie en 2015 en se basant sur la Dixième Révision de la Classification internationale des maladies (CIM-10).

Méthodes : il s'agissait d'une étude transversale et descriptive portant sur tous les décès autopsiés au nord de la Tunisie en 2015. Les caractéristiques socio-démographiques ont été identifiées, ainsi que les formes médico-légales et les causes de décès. Celles-ci ont été codées selon la CIM-10.

Résultats : notre échantillon était composé de 1957 cas. Il y avait une prédominance masculine avec un sex ratio de 3,5. Lâge moyen était de 47,2 (écart type [ET] 20,6) ans. Les morts violentes ont représenté $57,4 \%$ de la mortalité globale, suivies du groupe des maladies de l'appareil circulatoire $(24,5 \%)$ et de celui des maladies de l'appareil respiratoire (6,5\%). Les causes de décès les plus fréquentes étaient les accidents de transport terrestre $(33,7 \%)$ et les cardiopathies ischémiques (17,9\%). Les accidents de la voie publique étaient à l'origine de $56,1 \%$ des morts accidentelles. La pendaison était le mode suicidaire le plus fréquent (51,5\%). Les homicides étaient le plus souvent secondaires à des plaies par arme blanche $(35,8 \%)$.

Conclusion : la CIM-10 a permis de mettre en avant la part de la mort violente et ses caractéristiques dans la mortalité médico-légale au nord de la Tunisie, ce qui témoigne de son efficacité dans la standardisation des conclusions des rapports d'autopsie.

Mots clés : CIM-10, Tunisie, codage, mortalité, médico-légale.

Citation : Hmandi O; Ben Khelil M; Zoghlami N; Aounallah H; Hamdoun H. Apport de la dixième révision de la Classification internationale des maladies dans le codage de la mortalité médico-légale East Mediterr Health J. 2021;27(6):605-611. https://doi.org/10.26719/2021.27.6.605

Reçu : 01/08/19; accepté : 21/09/20

(c) Organisation mondiale de la Santé (OMS) 2021. Accès libre. Certains droits réservés. Ce document est disponible sous la licence CC BY-NC-SA 3.0 IGO. (https://creativecommons.org/licenses/by-nc-sa/3.0/igo)

\section{Introduction}

En Tunisie, le rapport de mortalité établi par l'Institut National de Santé Publique (INSP) n'inclut pas les causes de décès médico-légaux (1). Ce vide statistique peut être préjudiciable aux stratégies de prévention primaire du fait de l'absence de données fiables et officielles concernant les morts violentes ainsi que de celles relatives aux morts subites $(2,3)$.

En effet, la rédaction de certificats médicaux de décès $(C M D)$ par les médecins légistes après établissement des rapports d'autopsie n'est pas systématique en Tunisie. Dans la littérature scientifique, des facteurs expliquant cette «attitude » ont pu être mis en évidence, tels que la noncompréhension de la pertinence de cette pratique, la réticence par rapport au fait de refaire un acte médicoadministratif, le refus de lever l'obstacle médico-légal, la méconnaissance de l'intérêt en santé publique ou le manque de temps (4).

D'autre part, les rapports d'autopsie médico-légale en Tunisie ne se basent pas sur une classification telle que la dixième révision de la Classification internationale des maladies (CIM-10). Cela ne permet pas d'utiliser ces données de façon optimale et comparable dans l'établissement des rapports de mortalité. D'où l'importance d'établir un rapport de mortalité des décès médico-légaux en utilisant la CIM-10.

L'objectif de notre étude était d'établir les statistiques sur les causes de décès médico-légaux au nord de la Tunisie en 2015 en se basant sur la CIM-10.

\section{Méthodes}

Nous avons réalisé une étude transversale et descriptive portant sur tous les décès autopsiés au service de Médecine légale de l'hôpital Charles Nicolle de Tunis, survenus durant l'année 2015. Notre service couvre l'activité médico-légale de dix gouvernorats parmi les onze du nord de la Tunisie (à l'exception du gouvernorat de Nabeul), soit une population générale d'environ 4,56 millions d'habitants (5). La population de Nabeul représente 17,3\% de notre population d'étude et présente les mêmes caractéristiques démographiques que le reste des dix gouvernorats du Nord (5), nous 
permettant de supposer que nos résultats peuvent être représentatifs du nord de la Tunisie. Les données ont été recueillies à partir de celles de la réquisition judiciaire, de la copie du rapport d'autopsie médicolégale et, dans certains cas, des documents médicaux.

Dans ce travail, on s'est intéressé essentiellement aux caractéristiques socio-démographiques des défunts, à la forme médico-légale et à l'enchainement causal du décès tel que défini dans le certificat médical de décès tunisien, similaire à celui du modèle international élaboré par l'Organisation mondiale de la Santé (OMS). Dans un deuxième temps, les causes de décès ont été codées selon la dixième révision de la Classification internationale des maladies (CIM-10). Les statistiques de mortalité ont porté sur la cause initiale.

Nous avons choisi que le codage soit réalisé par un médecin légiste. La cause de décès a été établie en se basant sur la synthèse des éléments relevés des documents médicaux ainsi que de l'autopsie médicolégale. Dans le cas où l'autopsie ne permettait pas de conclure avec certitude par rapport à la cause de décès, celle-ci était codée R99 « Autres causes de mortalité mal définies et non précisées ».

Les données ont été traitées à l'INSP à l'aide du logiciel Microsoft Excel 2007 et du logiciel SPSS version 20.0 pour Windows.

\section{Résultats}

En 2015, 65743 décès sont survenus en Tunisie. Notre échantillon était composé de 1957 cas représentant un ratio de mortalité médico-légale de 6,6\%. Il y avait une prédominance masculine avec un sex ratio de 3,5. L'âge moyen était de 47,2 (ET 20,6) ans, avec des extrêmes d'un jour et de 95 ans. Ces cas étaient principalement mariés (52,3\%) ou célibataires (39,3\%). La majorité des défunts étaient décédés à Tunis (41,6\%). Toutes les causes de décès étaient documentées par les données de l'autopsie et des documents médico-légaux. Au total $5,4 \%$ des cas sont restés sans cause de décès exacte (Figure 1).

Le groupe des « causes externes de morbidité et de mortalité » (V01-Y98) était le premier groupe de causes de mortalité dans notre échantillon avec 1018 cas (52,0 \%), suivi des «maladies de l'appareil circulatoire 》 (I00-I99) (24,5\%). Les «lésions traumatiques, empoisonnements et certaines autres conséquences de causes externes » (S00-T98) sont classés en quatrième position (5,4\%). Ainsi, les morts violentes ont représenté $57,4 \%$ de la mortalité globale (Figure 2).

Les dix premières causes de mortalité formaient à elles seules 70,1 \% des causes de mortalité avec 1372 cas. Les causes les plus fréquentes étaient les accidents de transport terrestre (V01-V89) (33,7 \%) et les cardiopathies ischémiques (I20-I25) (17,9\%) (Figure 1).

Les morts accidentelles étaient la forme médicolégale la plus fréquente $(41,7 \%)$, suivie des morts naturelles $(31,6 \%)$ et des formes médico-légales indéterminées (13\%). Les suicides et les homicides représentaient respectivement $8,3 \%$ et $5,4 \%$ des décès. Les accidents de la voie publique étaient responsables de $56,1 \%$ des morts accidentelles; les piétons étaient les victimes les plus atteintes (44,4\%). Parmi les huit modes suicidaires qui ont été observés, la pendaison était responsable à elle seule de $51,5 \%$ du total des suicides. Il y avait 106 cas d'homicide, le plus souvent secondaire à des plaies par arme blanche (35,8\%) et à des blessures par objet contondant $(27,4 \%)$. Nous avons colligé aussi 13 décès secondaires à un blast à haute énergie (explosion) (12,3\%) et neuf cas de blessure par arme à feu à issue fatale $(8,5 \%)$ en rapport avec des attentats terroristes.

Tableau 1 Distribution des groupes causes de décès selon le sexe

\begin{tabular}{|c|c|c|c|c|}
\hline \multirow[t]{2}{*}{ Principaux groupes de maladies ou causes extérieures (Code CIM-10) } & \multicolumn{2}{|c|}{ Hommes } & \multicolumn{2}{|c|}{ Femmes } \\
\hline & Nbre & $\%$ & Nbre & $\%$ \\
\hline $\begin{array}{l}\text { Causes externes de morbidité et de mortalité } \\
\text { (V01-Y98) }\end{array}$ & 821 & 53,7 & 197 & 46,1 \\
\hline Maladies de l'appareil circulatoire (I00-I99) & 384 & 25,1 & 95 & 22,2 \\
\hline Maladies de l'appareil respiratoire (J00-J99) & 97 & 6,3 & 30 & 7,0 \\
\hline $\begin{array}{l}\text { Symptômes, signes et résultats anormaux d'examens cliniques et de laboratoire, non } \\
\text { classés ailleurs (R00-R99) }\end{array}$ & 65 & 4,3 & 34 & 8,0 \\
\hline $\begin{array}{l}\text { Lésions traumatiques, empoisonnements et certaines autres conséquences de causes } \\
\text { externes (S00-T98) }\end{array}$ & 78 & 5,1 & 27 & 6,3 \\
\hline Maladies de l'appareil digestif (K00-K93) & 22 & 1,4 & 8 & 1,8 \\
\hline Tumeurs (C00-D48) & 18 & $1,2 \%$ & 8 & $1,9 \%$ \\
\hline Maladies endocriniennes, nutritionnelles et métaboliques (E00-E90) & 11 & 0,7 & 11 & 2,6 \\
\hline Maladies du système nerveux (G00-G99) & 12 & 0,8 & 5 & 1,2 \\
\hline Autres causes & 22 & 1,4 & 12 & 2,8 \\
\hline Total & 1530 & 100 & 427 & 100 \\
\hline
\end{tabular}




\begin{tabular}{|c|c|c|c|c|c|c|c|}
\hline \multirow[t]{2}{*}{ Causes } & \multicolumn{7}{|c|}{ Âge (ans) } \\
\hline & $\begin{array}{c}<15 \\
(n=115)\end{array}$ & $\begin{array}{c}15-24 \\
(n=163)\end{array}$ & $\begin{array}{l}25-34 \\
(n= \\
280)\end{array}$ & $\begin{array}{c}35-44 \\
(n= \\
269)\end{array}$ & $\begin{array}{c}45-54 \\
(n=337)\end{array}$ & $\begin{array}{c}55-64 \\
(\mathrm{n}= \\
358)\end{array}$ & $\begin{array}{l}\geq 65 \\
(\mathrm{n}= \\
406)\end{array}$ \\
\hline $\begin{array}{l}\text { Causes externes de morbidité et de mortalité } \\
\text { (V01-Y98) }\end{array}$ & $62,6 \%$ & $73,0 \%$ & $70,4 \%$ & $58,7 \%$ & $42,1 \%$ & $35,5 \%$ & $45,6 \%$ \\
\hline Maladies de l'appareil circulatoire (I00-I99) & $1,7 \%$ & $9,9 \%$ & $11,4 \%$ & $11,9 \%$ & $31,8 \%$ & $41,3 \%$ & $35,0 \%$ \\
\hline Maladies de l'appareil respiratoire (J00-J99) & $9,6 \%$ & $0,6 \%$ & $2,5 \%$ & $8,2 \%$ & $6,5 \%$ & $7,8 \%$ & $8,1 \%$ \\
\hline $\begin{array}{l}\text { Symptômes, signes et résultats anormaux d'examens } \\
\text { cliniques et de laboratoire, non classés ailleurs } \\
\text { (R00-R99) }\end{array}$ & $10,4 \%$ & $1,8 \%$ & $2,5 \%$ & $6,3 \%$ & $6,8 \%$ & $5,3 \%$ & $2,5 \%$ \\
\hline $\begin{array}{l}\text { Lésions traumatiques, empoisonnements et certaines } \\
\text { autres conséquences de causes externes (S00-T98) }\end{array}$ & $6,2 \%$ & $9,8 \%$ & $8,6 \%$ & $8,2 \%$ & $3,0 \%$ & $3,6 \%$ & $3,2 \%$ \\
\hline Maladies de l'appareil digestif (K00-K93) & $2,6 \%$ & $0,6 \%$ & $0,0 \%$ & $1,1 \%$ & $2,4 \%$ & $1,4 \%$ & $2,5 \%$ \\
\hline Tumeurs (C00-D48) & $0,0 \%$ & $0,6 \%$ & $0,0 \%$ & $1,5 \%$ & $2,6 \%$ & $2,0 \%$ & $1,2 \%$ \\
\hline $\begin{array}{l}\text { Maladies endocriniennes, nutritionnelles et } \\
\text { métaboliques (E00-E90) }\end{array}$ & $1,7 \%$ & $0,6 \%$ & $1,4 \%$ & $0,7 \%$ & $1,8 \%$ & $1,1 \%$ & $0,7 \%$ \\
\hline Maladies du système nerveux (G00-G99) & $0,9 \%$ & $0,6 \%$ & $1,8 \%$ & $1,5 \%$ & $1,5 \%$ & $0,3 \%$ & $0,0 \%$ \\
\hline Autres causes & $4,3 \%$ & $2,5 \%$ & $1,4 \%$ & $1,9 \%$ & $1,5 \%$ & $1,7 \%$ & $1,2 \%$ \\
\hline Total & $100 \%$ & $100 \%$ & $100 \%$ & $100 \%$ & $100 \%$ & $100 \%$ & $100 \%$ \\
\hline
\end{tabular}

${ }^{*}$ L'information relative à l'âge était manquante chez 29 cas.

Les « causes externes de morbidité et de mortalité » représentaient le groupe de mortalité le plus fréquent chez les deux sexes ( $53,7 \%$ des hommes et $46,1 \%$ des femmes) et les « maladies de l'appareil circulatoire » étaient la deuxième cause de mortalité $(25,1 \%$ des hommes et $22,2 \%$ des femmes) (Tableau 1). La mort violente était la première cause de décès pour toutes les tranches d'âge à l'exception de celle des 55-64 ans (Tableau 2).

\section{Discussion}

Dans notre étude, la mort violente explorée par les chapitres XIX et XX de la CIM-10 représentait à elle seule plus de la moitié des causes de décès (57,4\%). Cependant, elle ne représentait que $6,3 \%$ de la mortalité totale sur tout le territoire tunisien si on se référait au rapport national de mortalité de 2013 élaboré par l'INSP (1) et 3,8 \% par rapport à la mortalité totale dans les gouvernorats inclus dans notre étude (5). La mortalité en rapport avec les maladies de l'appareil circulatoire était observée au deuxième rang $(24,5 \%)$. Cependant, ce groupe de causes de décès représentait le groupe le plus fréquent à l'échelle nationale dans le rapport national de mortalité de $2013(29,1 \%)$ (1). La mortalité relative à la mort violente se rapprochait de celle d'une étude réalisée à Alger en 2014 (44,2 \%) (3). Elle était responsable de $63 \%$ des décès dans l'étude réalisée à Toulouse (6). $\mathrm{Au}$ Canada, la mort violente était la deuxième cause de mortalité après les causes médicales (35,5\%) (7). Les maladies cardio-circulatoires représentaient la deuxième cause de décès après la mort violente. Dans le contexte médico-légal, ce groupe de cause de décès est essentiellement observé dans le cadre de la mort subite d'origine cardiaque (MSC). Dans notre étude, la cardiopathie ischémique était la cause de décès la plus fréquente parmi les maladies cardio-vasculaires, suivie des «autres formes de cardiopathies » en référence principalement aux cardiomyopathies hypertrophiques, aux cardiomyopathies dilatées et aux dysplasies arythmogènes du ventricule droit. Nos résultats sont similaires aux résultats d'autres études médicolégales internationales $(8,9)$. Cette prédominance peut être expliquée par les mauvaises habitudes de vie, les facteurs de risque cardio-vasculaires mais aussi souvent par une négligence de la part des patients des signes fonctionnels pourvoyeurs de crise angineuse dont principalement la douleur thoracique, tous responsables d'un retard voire d'une absence de consultation et de prise en charge à temps (10).

Les piétons étaient les victimes les plus atteintes par les accidents de la circulation mortels dans notre étude. Selon l'Observatoire National de Sécurité Routière tunisien, ils étaient classés en troisième position $(25,87 \%)$ et les occupants d'une automobile étaient au premier rang (56,15\%) (11). À l'échelle internationale, les piétons venaient en deuxième position $(23 \%)$ après les occupants d'une automobile ( $28 \%$ ). En Afrique, $43 \%$ des victimes sont des piétons et des cyclistes, et dans le Sud-Est de l'Asie et sur la côte ouest du Pacifique, les motocyclistes et les conducteurs de véhicules à trois roues représentent la plus grande proportion (12). En effet, l'OMS définit les piétons, les cyclistes et les motocyclistes comme des utilisateurs de la route «vulnérables » à cause du manque de stratégies et d'outils de protection destinés à cette population contre une «favorisation» des 
Figure 1 Les dix principales causes de décès $(n=1372)$

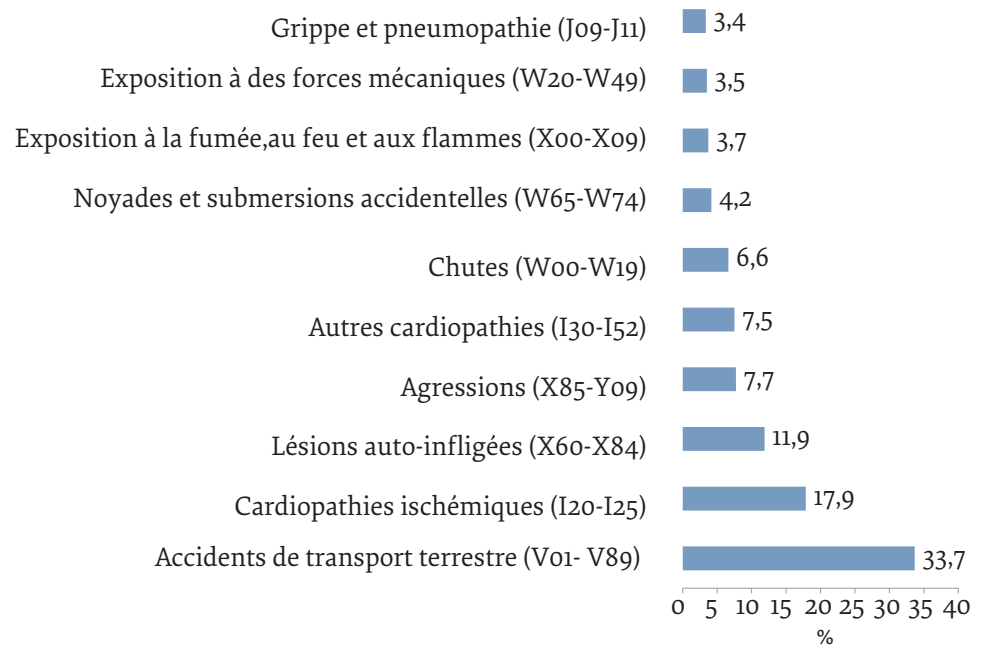

utilisateurs de véhicules à moteur dans l'usage de l'infrastructure et l'existence de moyens de protection dans les véhicules comme les airbags, la ceinture de sécurité, la stabilité du véhicule et la notification de dépassement d'une certaine vitesse (13). De plus, elle explique cette différence de répartition des victimes d'une région à une autre par la différence de recours aux différents moyens de transport et par le niveau socio-économique du pays. Par exemple, dans les pays à revenu faible ou moyen, le développement économique s'accompagne d'une croissance des moyens de transport motorisés mais l'infrastructure, les modalités de protection et de prise en charge des victimes des accidents de la voie publique, la législation et l'éducation routière ne se sont pas améliorées en parallèle $(13,14)$.
De nos résultats il ressort que la pendaison est le premier mode de suicide. Elle est aussi le mode suicidaire le plus fréquent dans les études traitant du suicide en Tunisie chez les adultes et les enfants (15,16). À Ankara également, elle est le mode le plus recouru chez les sujets âgés de plus de 65 ans (56,6 \%) (17). À Alger, elle est en deuxième position $(25,96 \%)$, légèrement dépassée par les précipitations (28,84\%) (3). Aux États-Unis d'Amérique, le suicide par asphyxie était classé deuxième après le suicide par arme à feu en 2014 et la pendaison était la méthode de suicide par asphyxie la plus utilisée dans 16 États de 2005 à 2014 (18).

Les plaies par arme blanche étaient les premières responsables de décès criminels en 2015 (35,8\%), suivies par les homicides secondaires à des agressions par objet contondant (27,4\%). Ce résultat rejoint celui

\section{Figure 2 Distribution des cas médico-légaux selon les groupes de décès}

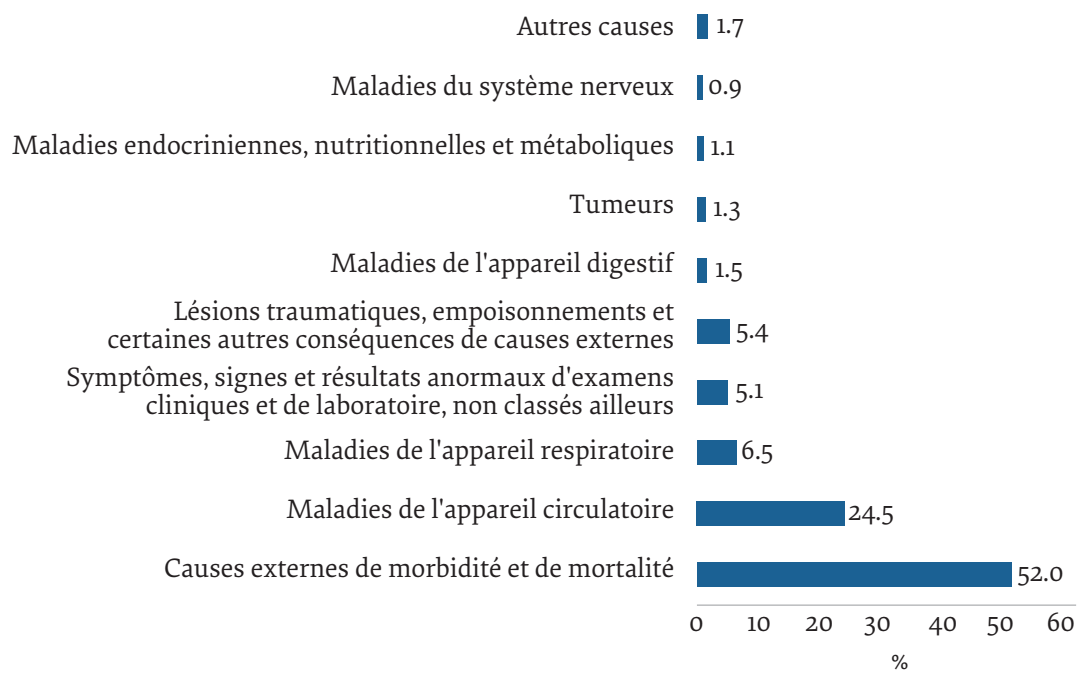


de deux études tunisiennes analysant cette forme médico-légale $(19,20)$. La particularité en 2015 dans notre étude était représentée par une augmentation des homicides par arme à feu et par blast à haute énergie (explosion d'engin), mais cette augmentation est à relativiser étant donné que ces cas étaient majoritairement survenus dans le cadre d'attentats terroristes ne reflétant pas un changement de modèle des homicides dans le cadre du droit commun. Il est plus fait usage d'armes à feu dans les homicides dans les pays occidentaux, en particulier aux États-Unis d'Amérique (21). Ce constat peut être expliqué par la différence d'accès aux armes dans les différents pays. En Tunisie, la législation régissant le port et l'usage des armes à feu est extrêmement restrictive, limitant l'accès aux armes à feu et privilégiant l'usage des armes blanches du fait de la facilité de se les procurer et de les utiliser tout en garantissant une issue fatale à l'agression.

Le choix qu'un médecin légiste fasse le codage était à notre avis utile afin de limiter les biais. Les données extraites des documents médico-légaux ne seraient pas disponibles aux codeurs du fait de leur caractère confidentiel et du contexte médico-judiciaire. Nous recommandons de garder cette démarche en pratique quotidienne afin de limiter les risques de codage non concluant.

Ce travail a aussi mis en évidence certaines difficultés et certaines limites en rapport avec la CIM10 elle-même. En effet, l'usage de causes de décès fonctionnelles dont les dysfonctions vitales telles que «insuffisance respiratoire aiguë», «insuffisance cardiaque aiguë», «œdème pulmonaire », sans pour autant remonter à la cause initiale bien qu'importante pour la pratique médico-légale, ne permettrait pas d'avoir un usage à visée épidémiologique. D'ailleurs, dans ce sens, l'OMS avait qualifié ces causes non spécifiques dans la CIM-10 de garbage codes puisqu'elles ne seront d'aucune utilité ni aux statistiques, ni dans l'élaboration des stratégies de prévention. En revanche, dans certains cas, le médecin légiste pourra identifier le mécanisme de décès ou éliminer d'autres mécanismes sans pour autant déterminer la cause exacte de décès. Exemple: «la mort est la conséquence d'un œdème pulmonaire. Elle n'est ni de cause traumatique ni de cause toxique ». L'essentiel est donc de garantir la qualité des rapports d'autopsie au sens médico-légal tout en gardant la possibilité d'utiliser ces rapports (ou les CMD remplis a posteriori) dans les travaux visant à protéger la santé publique.

Une autre limite de l'usage de la CIM-10 était son caractère mono-axial ne permettant pas de coder plus d'une cause initiale. En effet, même le système de dague ou d'astérisque garantissant une double classification n'était pas efficace dans le contexte médico-légal. On peut citer l'exemple d'une agression avec de multiples agresseurs où le légiste devrait s'efforcer d'identifier les différents traumatismes ayant pu aboutir au décès et de discuter l'imputabilité de chacune des lésions provoquées par chacun des agresseurs. Au moment de coder la cause initiale de décès, le médecin légiste devrait choisir laquelle des causes/lequel des mécanismes à retenir, qui est à l'origine de l'enchainement causal. Dissocier les données du rapport d'autopsie médico-légale destiné à l'autorité judiciaire d'une part, du certificat médical de décès rempli a posteriori et délivré seulement à l'autorité sanitaire responsable du codage d'autre part, s'avère ainsi primordial. Le médecin légiste pourrait procéder lui-même au codage afin d'adapter le message à chacune des parties prenantes.

En conclusion, notre étude a permis d'établir les statistiques sur les causes de décès médico-légaux, en particulier la part de la mort violente et ses caractéristiques. La CIM-10 s'est avérée un moyen utile de standardisation des conclusions des rapports d'autopsie. Nos résultats pourraient représenter les prémices d'une rubrique au titre de «Mortalité médico-légale» dans le rapport de mortalité de la population générale des dix gouvernorats du nord de la Tunisie couverts par notre service. L'usage de la CIM10 pouvait présenter des difficultés, d'où limportance d'un entrainement préalable et d'une proposition d'une version de la CIM-10 adaptée à l'activité médico-légale permettant d'optimiser le codage et donc l'usage dans les interventions visant la santé publique.

Conflits d'intérêt : aucun

Financements : aucun

\title{
Contribution of 10th Revision of the International Classification of Diseases to the Coding of Medico-Legal Mortality
}

\author{
Abstract \\ Background: Tunisian official mortality reports do not include causes of medico-legal death. \\ Aims: Establish the medico-legal death statistics of Northern Tunisia according to the 1oth version of the International \\ Classification of Diseases (ICD-10). \\ Methods: A cross-sectional descriptive review, including all medico-legal deaths investigated in Northern Tunisia in 2015. \\ Data related to the sociodemographic features were collected. Causes and manner of death were coded according to the \\ ICD-10.
}


Results: We included 1957 cases. Victims were mainly males. Sex ratio (M/F) was 3.5 with a mean age of $47.2 \pm 20.6$ years. Violent death represented $57.4 \%$ of the total sample, diseases of the circulatory system and of the respiratory systems counted respectively for $24.5 \%$ and $6.5 \%$. Road traffic accidents were the cause of $56.1 \%$ of accidental deaths. Hanging was the most frequent suicidal mode (51.5\%). Homicides were most frequently caused by sharp force (35.8\%).

Conclusion: ICD-10 was a useful tool to code medico legal death and to highlight violent death causes in the population mortality report in Northern Tunisia. This would be helpful to enable a standardization of autopsy reports aligned with a public health use of these data.

$$
\text { أنس حساندمة المر اجعة العاشرة للتصنيف الدولي للأمر اض في ترميز الوفيات التي نظر فيها الطب الشرعي }
$$

$$
\text { الخلفية: لا تتضمن تقارير الوفيات الرسمية التونسية أسباب الوفيات التي ينظر فيها الطب الشرعي. }
$$

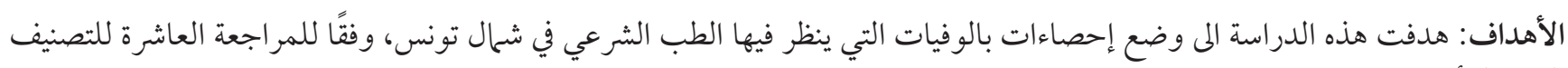

$$
\text { الدولي للأمراض: هدف. }
$$

طرق البحث: إجراء مراجعة وصفية مقطعية، تتضمن جميع الوفيات التي ينظر فيها الطب الشرعي، والتي جرى التحق التحقيق بشأنها في شهال تونس في

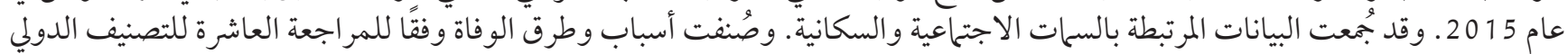
لأمر اض.

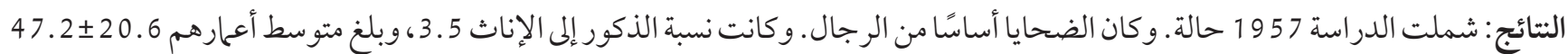

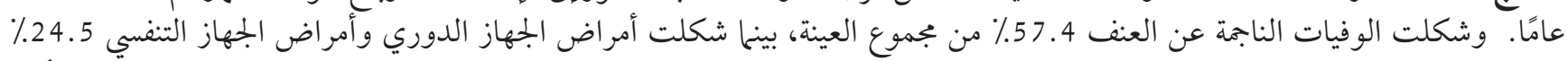

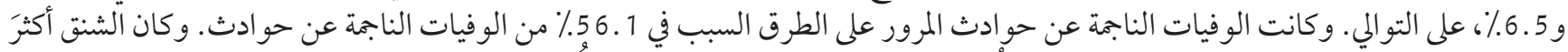

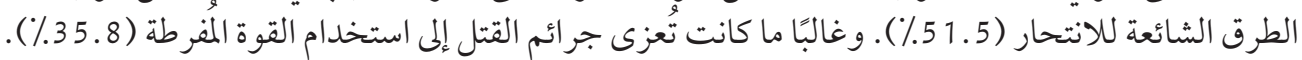

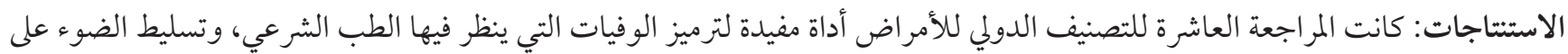

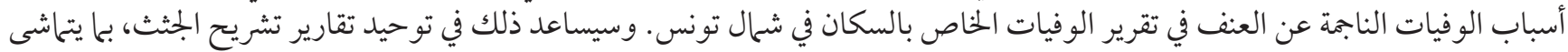
مع استخدام الصحة العامة لهذه البيانات.

\section{Références}

1. Hajem S. Le système national d’information sur les causes médicales de décès -Entraves spécifiques, synthèse des principaux résultats de l'année 2013 et perspectives. Tunis: Institut National de Santé Publique (INSP), Unité de Recherche sur le Vieillissement et les Causes Médicales de Décès; mars 2015.

2. Richaud-Eyraud E, Rondet C, Rey G. Transmission des certificats de décès au CépiDc-Inserm en cas de mort suspecte en France, depuis 2000. Rev Epidemiol Santé Publique. 2018;66(2):125-33. https://doi.org/10.1016/j.respe.2017.11.006

3. Achiou D. Apport de l'activité médico-légale pour la statistique des causes de décès dans la wilaya d'Alger [thèse]. Alger, Faculté de Médecine d'Alger, 2016 (http://biblio.univ-alger.dz/jspui/bitstream/1635/14380/1/ACHIOU_DJAMAAL.pdf, consulté le 21 janvier 2021).

4. CépiDC-Inserm et Santé publique France. Enquête sur les activités et pratiques des instituts médico-légaux en France, en 2016, en vue de l'amélioration des connaissances des données sur la mortalité. In: Observatoire national du suicide (ONS). Suicide : enjeux éthiques de la prévention, singularités du suicide à l'adolescence - 3e rapport; 2018: 197-201 (https://drees. solidarites-sante.gouv.fr/IMG/pdf/fiche7-10.pdf ons3.pdf (solidarites-sante.gouv.fr), consulté le 28 janvier 2021).

5. Population [Internet]. Tunis: Institut National de la Statistique (http://www.ins.tn/fr/themes/population, consulté le 21 janvier 2021).

6. Savall F, Dédouit F, Gainza D, Blanc A, Costagliola R, Telmon N, et al. Épidémiologie des décès médicolégaux : une nécessité ? Rev Med Legal. 2013 Mars;4(1):20-6. https://doi.org/10.1016/j.medleg.2012.11.002

7. Base canadienne de données des coroners et des médecins légistes [Internet]. Rapport annuel 2006 à 2008. Ottawa: Statistique Canada, 2012;6-11 (http://www.statcan.gc.ca/pub/https://www150.statcan.gc.ca/n1/pub/82-214-x/82-214-x2012001fra.pdf, consulté le 21 janvier 2021).

8. Mazzanti A, O’Rourke S, Ng K, Miceli C, Borio G, Curcio A, et al. The usual suspects in sudden cardiac death of the young: a focus on inherited arrhythmogenic diseases. Expert Rev Cardiovasc Ther. 2014 Apr;12(4):499-519. https://doi.org/10.1586/1477 9072.2014.894884 PMID:24650315

9. Ding Z, Yang M, Wang Y, Wu S, Qiu X, Liu Q. Retrospective analysis of 769 cases of sudden cardiac death from 2006 to 2015 : a forensic experience in China. Forensic Sci Med Pathol. 2017 Sep;13(3):336-41. https://doi.org/10.1007/s12024-017-9888-z PMID:28752200 
10. Mesrati MA, Belhadj M, Aissaoui A, HajSalem N, Oualha D, Boughattas M, et al. La mort subite cardiovasculaire de l'adulte : étude autopsique de 361 cas. Ann Cardiol Angeiol. 2017 Feb;66(1):7-14. https://doi.org/10.1016/j.ancard.2016.03.003

11. Statistiques des accidents de la circulation. Répartition des accidents selon les victimes 2015. Tunis: Observatoire National de la Sécurité Routière (ONSR) [en arabe] ( http://onsr.nat.tn/onsr/pdf/2015.pdf, consulté le 9 février 2021).

12. Global status report on road safety 2015. Geneva: World Health Organization; 2015 (https://www.who.int/violence_injury_ prevention/road_safety_status/2015/en/, consulté le 21 janvier 2021).

13. Global status report on road safety 2018. Geneva: World Health Organization; 2018 (https://www.who.int/violence_injury_ prevention/road_safety_status/2018/en/, consulté le 21 janvier 2021). https://www.who.int/publications/i/item/9789241565684

14. Performance Assessment Report 2012-2013 - Appendix: OWER indicators and Detailed Outcome Assessment Sheets. Background documents. Sixty-fourth session of the Regional Committee for Europe, Copenhagen, Denmark, 15-18 September 2014. Copenhagen: World Health Organization, Regional Office for Europe; 2014, p. 117 ( https://www.euro.who.int/fr/ about-us/governance/regional-committee-for-europe/past-sessions/64th-session/documentation/background-documents, consulté le 9 février 2021).

15. Majdoub W, Mosbahi A, Naouar M, Beji M, Mannai J, Turki E. Suicide in children and adolescents: a Tunisian perspective from 2009 to 2015. Forensic Sci Med Pathol. 2017 Dec;13(4):417-25. https://doi.org/10.1007/s12024-017-9909-y PMID:28879455

16. Ben Khelil M, Zgarni A, Zaafrane M, Chkribane Y, Gharbaoui M, Harzallah H, et al. Suicide by self-immolation in Tunisia: A 10 year study (2005-2014). Burns. 2016 Nov;42(7):1593-9. https://doi.org/10.1016/j.burns.2016.04.019 PMID:27211362

17. Akar T, Karapirli M, Akcan R, Demirel B, Akduman B, Dursun AZ, et al. Elderly deaths in Ankara, Turkey. Arch Gerontol Geriatr. 2014 Sep-Oct;59(2):398-402. https://doi.org/10.1016/j.archger.2014.05.005 PMID:24962235

18. Yau RK, Paschall MJ. Epidemiology of asphyxiation suicides in the United States, 2005-2014. Inj Epidemiol. 2018 Jan 8;5(1):1-9. https://doi.org.10.1186/s40621-017-0131-x PMID:29308538

19. Ben Khelil M, Farhani F, Harzallah H, Allouche M, Gharbaoui M, Banasr A, et al. Patterns of homicide in North Tunisia: a 10-year study (2005-2014). Inj Prev. 2018 Feb 9;24(1):73-7. https://doi.org/10.1136/injuryprev-2016-042123

20. Ben Khelil M, Gharbaoui M, Farhani F, Zaafrane M, Harzallah H, Allouche M, et al. Impact of the Tunisian Revolution on homicide and suicide rates in Tunisia. Int J Public Health . 2016 Dec;61(9):995-1002. https://doi.org/10.1007/s00038-016-08348 PMID:27193575

21. Grinshteyn E, Hemenway D. Violent death rates in the US compared to those of the other high-income countries, 2015. Prev Med. 2019 Jun;123:20-6. https://doi.org/10.1016/j.ypmed.2019.02.026 